\title{
Observation of the TESPEL-injected impurities behaviour by the PHA system at Wendelstein 7-X
}

\author{
M. Kubkowska, ${ }^{a, 1}$ N. Tamura, ${ }^{b, c}$ A. Chomiczewska, ${ }^{a}$ T. Fornal, ${ }^{a}$ M. Gruca, ${ }^{a}$ N. \\ Krawczyk, ${ }^{a}$ S. Jabłoński, ${ }^{a} L$.' Ryć, ${ }^{\text {a }}$ R. Bussiahn, ${ }^{d}$ K. Brunner, ${ }^{d}$ R. Burhenn, ${ }^{d} J$. \\ Knauer, ${ }^{d} A$. Langenberg, ${ }^{d} U$. Neuner, ${ }^{d} H$. Thomsen ${ }^{d}$ and the $W 7-X$ team \\ ${ }^{a}$ Institute of Plasma Physics and Laser Microfusion, Hery 23, 01-497 Warsaw, Poland \\ ${ }^{b}$ National Institute for Fusion Science, National Institutes of Natural Sciences, 509-5292 Toki, Japan \\ ${ }^{c}$ SOKENDAI (The Graduate University for Advanced Studies), 509-5292 Toki, Japan \\ ${ }^{d}$ Max Planck Institute for Plasma Physics, 17491 Greifswald, Germany \\ E-mail: monika.kubkowska@ipplm.pl
}

\begin{abstract}
The Pulse Height Analysis (PHA) system at Wendelstein 7-X (W7-X) is a broad energy range spectrometer dedicated to the observation of impurity behaviour during the plasma discharges. The system measures spectra in the range between 0.5 and $20 \mathrm{keV}$, from integrated signals along three lines of sight that pass close to the plasma center. During the recent operational phase of W7-X, the TESPEL (Tracer-Encapsulated Solid PELlet) injector has been commissioned. By this injector, polystyrene pellets with a single tracer impurity as well as multiple tracer impurities have been injected into the W7-X plasmas. The multiple tracer impurities injection allows studying the behavior of various impurities in the same plasma condition. The time resolution of the PHA system in the order of $60-100 \mathrm{~ms}$, is smaller than typical confinement times and sufficient to observe differences in temporal behaviour of injected impurities. The collected PHA spectra consist of $\mathrm{K} \alpha$ lines of vanadium, manganese and nickel in case of the triple tracers, and of iron or copper in case of the single tracer. The temporal evolution of these $\mathrm{K}_{\alpha}$ lines has been obtained in various plasma conditions, which will be presented and discussed in this paper.
\end{abstract}

KEYWORDS: pulse height analyser; stellarator; solid pellet

\footnotetext{
${ }^{1}$ Corresponding author
} 


\section{Contents}

1. Introduction $r$

2. TESPEL and PHA systems at W7-X 2

3. PHA experimental results $r$

4. Summary $\quad 5$

\section{Introduction}

Wendelstein 7-X (W7-X) is a stellarator with superconducting magnetic field coils [1-2] that started its operation at the end of 2015. The first operation phase (OP1.1) of W7-X was performed with five graphite limiters mounted on the inboard side, which define the last closed flux surface (LCFS). During the first experimental campaign, commissioning of installed diagnostic systems and device components was performed [3]. All results obtained in the limiter configuration exceeded the expectations for the first experimental programs, at the same time delivering valuable material for physics investigations [4]. After OP1.1, W7-X restarted its work with graphite heat shields and ten inertially cooled island divertor modules. One of the main functions of island divertor is to control the interaction between plasma and the first wall and minimize an impurity influx. As a result, this particular setup in combination with a boronization of the first wall allows for W7-X high-performance plasma operation [5].

Understanding of impurity transport in stellarator plasmas is a key issue for proper optimisation of the device. Possible accumulation of heavy ion impurities may lead to undesired effects like decreased plasma performance or even early pulse termination [6]. It is also known that impurity confinement in plasma is mainly determined by transport mechanisms that occur in plasma. Therefore, it is particularly important to perform experiments dedicated to study the impurity transport in the core plasma. During the latest operational phase (OP1.2b) of W7-X, a Tracer-Encapsulated Solid Pellet (TESPEL) system has been commissioned and tested [7]. This system was developed in collaboration between CIEMAT (Spain), IPP-Greifswald (Germany) and NIFS (Japan). The TESPEL can release impurities at a well-localized radial position directly in the core plasma. Such a system was already deployed at LHD [8-9] and TJ-II [10]. Owing to its capability of containing multiple tracers inside the TESPEL, the system offers possibility of comparing the behaviour of various impurities exactly in the same plasma condition. The study of injected impurities behaviour is usually done by the main W7-X impurity diagnostics like High Efficiency XUV Overview Spectrometer (HEXOS) [11], X-ray Imaging Crystal Spectrometers (XICS) [12] and Pulse Height Analysis system (PHA) [13-14]. These diagnostics make observation of the temporal evolution of various emission lines from highly-ionized tracer impurities. In this paper, spectra observed by the broad energy range (up to $20 \mathrm{keV}$ ) PHA system are presented and discussed. 


\section{TESPEL and PHA systems at W7-X}

During the OP1.2b of W7-X, the TESPEL injector has been commissioned and tested. The injector was installed at Port AEE41 (see figure 1). The TESPEL is injected by using highpressured helium gas (pneumatic pipe-gun method) towards the plasma centre. In general, TESPEL has a spherical shape and consists of polystyrene as an outer layer with tracer impurities inside. Its diameter ranges between 700 and $900 \mu \mathrm{m}$. One of the advantages of TESPEL is that well-known amount of impurities can be deposited in plasma. A relatively wide range of different materials can be injected as a tracer in the TESPEL, which can be detected through line emissions in the VUV and soft X-ray region. In the W7-X experiments, the TESPEL with single- and tripletracer/s has been injected into the plasmas. In case of single tracer, copper $(\mathrm{Cu})$, tungsten $(\mathrm{W})$ or iron $(\mathrm{Fe})$ were used, while in case of triple-tracers, vanadium $(\mathrm{V})$, manganese $(\mathrm{Mn})$ and nickel (Ni) were applied. For all these elements, He-like ion lines emission can be observed by the PHA system $[15,16]$. The diagnostic delivers information about the impurity content based on the line identification and the temporal evolutions of emission lines with a time resolution of 50-100 ms. Thanks to the broader observable energy range of the PHA system [16], it can compare signals which originated from elements with different atomic numbers in the same experimental condition.

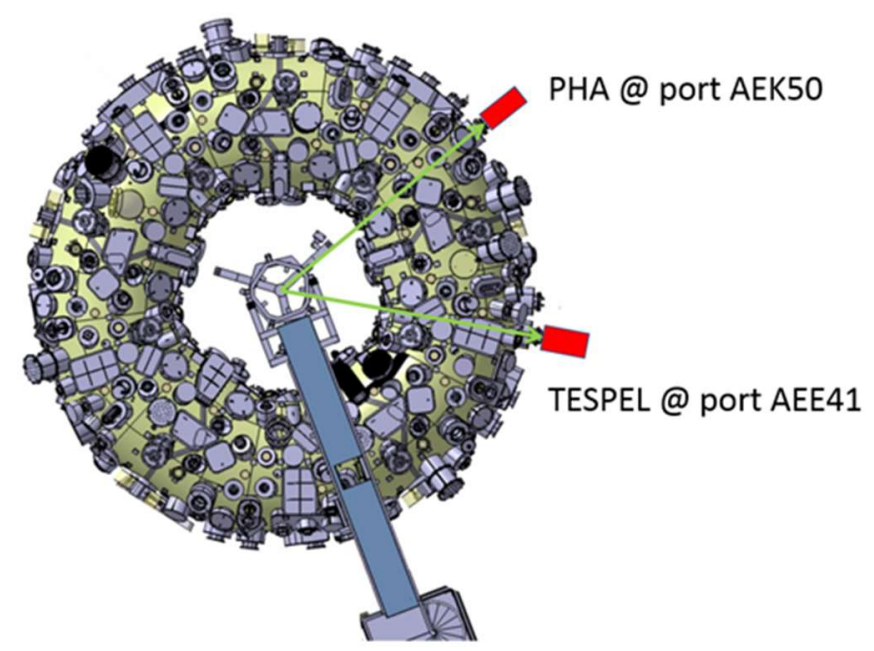

Figure 1. Location of the PHA and TESPEL systems at W7-X.

During the OP1.2b of W7-X, the first PHA channel, which is dedicated for the observation of line emissions from the medium- and high-Z elements, was equipped with an additional $1 \mathrm{~mm}$ thick Be foil to cover the energy range from 3.6 to $20 \mathrm{keV}$ (assuming $10 \%$ of transmission). The adjustable slits with a square shape, which regulate the flux of radiation reaching the detectors, were set from 800 to $1200 \mu \mathrm{m}$ according to experimental conditions.

\section{PHA experimental results}

Figure 2a shows the example of experimental conditions of discharge with the TESPEL injection. In this case, the TESPEL injection was done during the 3.5 MW ECRH with NBI blips. Figure $2 \mathrm{~b}$ shows collected spectra during the discharges with the TESPEL with triple tracer impurities 
(V, Mn, and $\mathrm{Ni}$ ) and single tracer impurity $(\mathrm{Cu})$. In both cases, He-like ion lines from the tracer impurities are clearly seen (Argon, which originated from gas-puff, is also observed). The energy resolution of collected spectra in these cases was $185 \mathrm{eV}$ for He-like Mn line at $6.180 \mathrm{keV}$. Due to an effective particle deposition using the TESPEL injector, the temporal evolution of intense spectral lines could be well resolved with the PHA system (time resolution of $100 \mathrm{~ms}$ ).
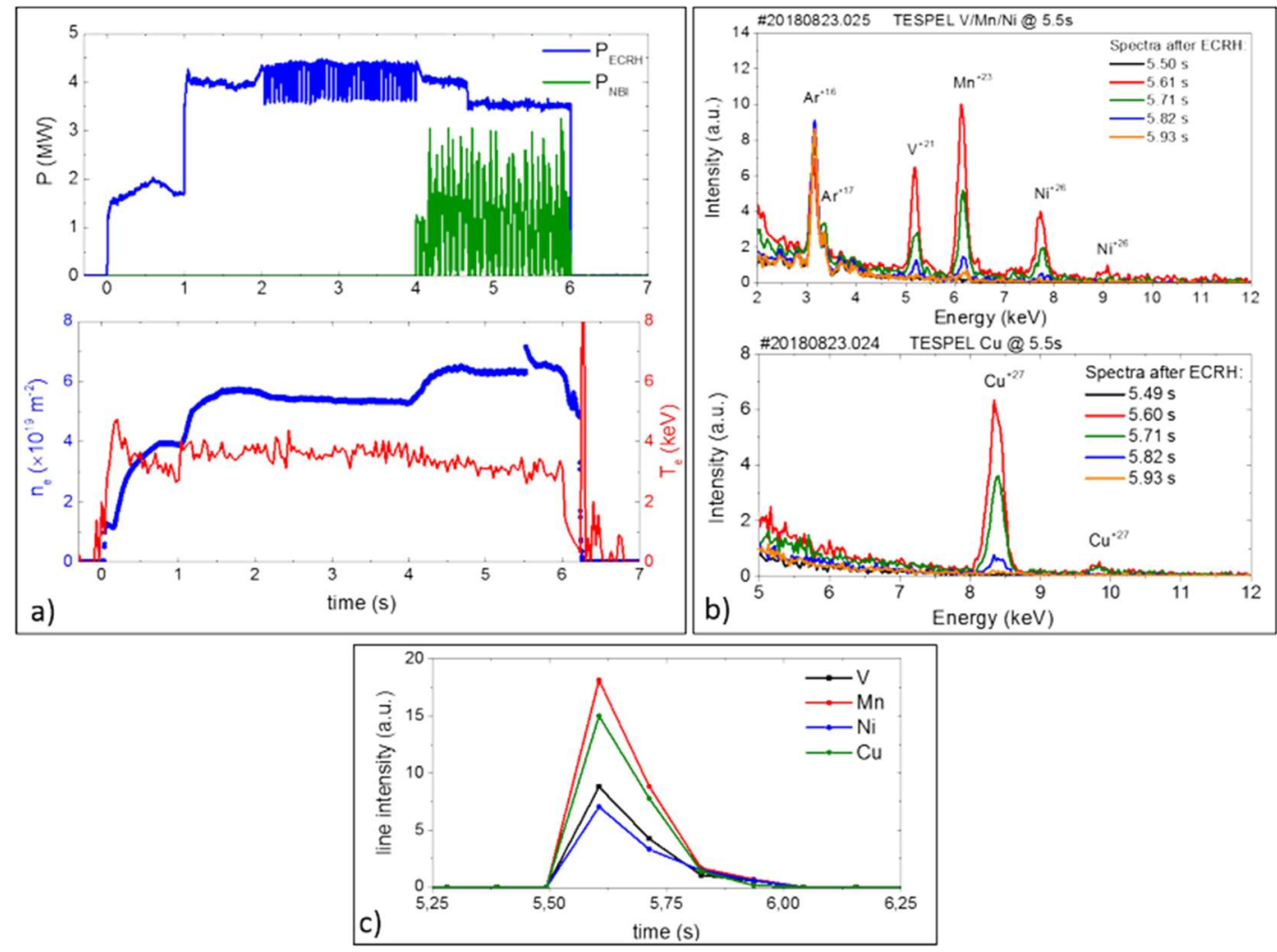

Figure 2. (a) Time traces of ECRH and NBI heating power (upper), electron density and temperature (lower) of the described W7-X discharge with TESPEL injection at $5.5 \mathrm{~s}$; (b) PHA spectra observed during the triple TESPEL of $\mathrm{V} / \mathrm{Mn} / \mathrm{Ni}$ (upper) and $\mathrm{Cu}$ (lower) injection; (c) time traces of injected impurities obtained from the PHA spectra.

The TESPEL with the triple tracer impurities allowed studying the behaviour of elements with different atomic numbers in the same plasma condition. Figure $2 \mathrm{c}$ shows time evolution of observed spectral lines depicted in figure $2 b$. Detailed investigation and results obtained by diagnostics with higher temporal resolution will be a subject of separate scientific paper.

An interesting behaviour was observed in the discharge with 1MW ECRH and NBI blips (see figure 3). In this discharge, the TESPEL containing silicon ( $\mathrm{Si}$ ) and iron (Fe) impurities was injected into plasma at $10.5 \mathrm{~s}$ with $\mathrm{Si}$ amount of $6.8 \times 10^{16}$ particles and $\mathrm{Fe}$ amount of $1.7 \times 10^{17}$ particles. It is worth adding that only a He-like iron line was observed in the spectra, which could suggest that the number of Si particles was not sufficient or the dominant charge-state of Si impurities did not manifest itself in the energy range observed by the PHA (Si lines were observed in none of the 3 energy PHA channels in the described discharge; normally $\mathrm{He}$ - and $\mathrm{H}$ like Si lines were observed in the spectra collected by the second channel at 1.86 and $2.00 \mathrm{keV}$ respectively [16]). Thanks to the appropriate diagnostic settings, the time resolution of the PHA was able to be set to $60 \mathrm{~ms}$ (see figure $3 \mathrm{c}$ ). After the TESPEL injection, the intensity line of $\mathrm{Fe}^{24+}$ 
at $6.7 \mathrm{keV}$ was kept at a certain level, while after switching off the NBI blips, a sudden decay of $\mathrm{Fe}^{24+}$ emission line was observed. Just after the additional effective heating power by the NBIblips is turned off, also electron temperature, $\mathrm{T}_{\mathrm{e}}$ drops immediately from about $1 \mathrm{keV}$ to $0.6 \mathrm{keV}$, and hence the ionization and excitation rates at these low temperatures will also drop significantly. In the case shown in figure 3, the line-integrated electron density after the TESPEL injection was about $6 \times 10^{19} \mathrm{~m}^{-2}$ and it was a bit decreased after switching off of NBI heating. In the LHD (Large Helical Device) stellarator, the accumulation of the impurities has been observed in a high-density regime [17]. In order to confirm similarities further investigation is needed.

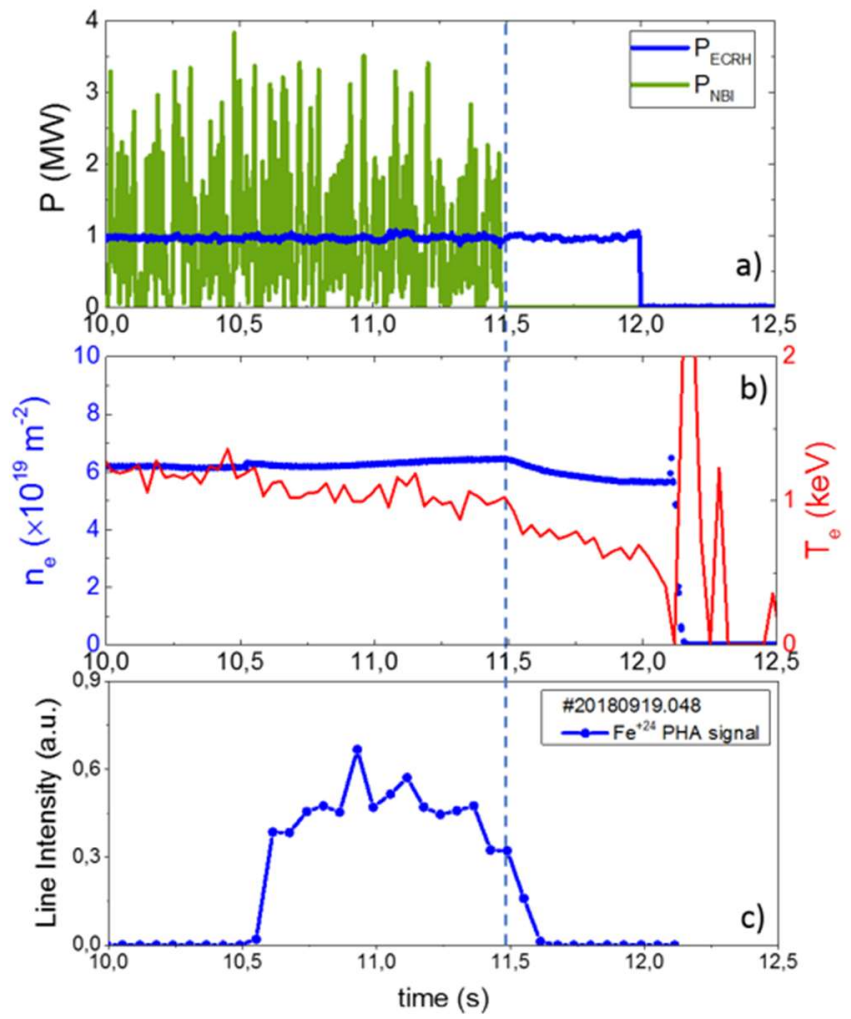

Figure 3. Temporal evolutions of (a) ECR and NBI heating; (b) the line-integrated electron density and temperature; (c) the temporal evolution of He-like iron line observed by the PHA system in 20180919.048 W7-X experimental programme. Dashed line corresponds to terminating the NBI heating.

Figure 4 shows another example of the discharge with the TESPEL having the triple tracer impurities. A long decay time of $\mathrm{K}_{\alpha}$ emission from the injected impurities was also observed in the discharge with the ECRH power of $3 \mathrm{MW}$ and the line-integrated electron density of about $7 \times 10^{19} \mathrm{~m}^{-2}$. In this case, the temporal behaviours of the $\mathrm{K}_{\alpha}$ emission were changed quickly probably due to the increment of ECRH power. After reducing the ECRH level from 4 to $3 \mathrm{MW}$ (5s) the temperature starts to rise slightly from about 1.5 to $2.2 \mathrm{keV}$ at $5.8 \mathrm{~s}$. This temperature rise will also steadily increase of $\mathrm{V}, \mathrm{Mn}$ and $\mathrm{Ni}$ signals in this time interval producing a longer decay time. In order to reveal the cause of such a sudden change of temporal behaviours of the $\mathrm{K}_{\alpha}$ emission, more detailed analysis is required, especially given the fact that the electron density in that case does not show any changes (figure $4 \mathrm{~b}$ ). From 5.8 till $6.2 \mathrm{~s}$ the electron temperature drops a little from 2.2 to $1.8 \mathrm{keV}$, and some part of the decay is probably due to this fact. The dependence on the additional ECRH power will be a subject of the scientific proposal for next W7-X experimental campaign. 

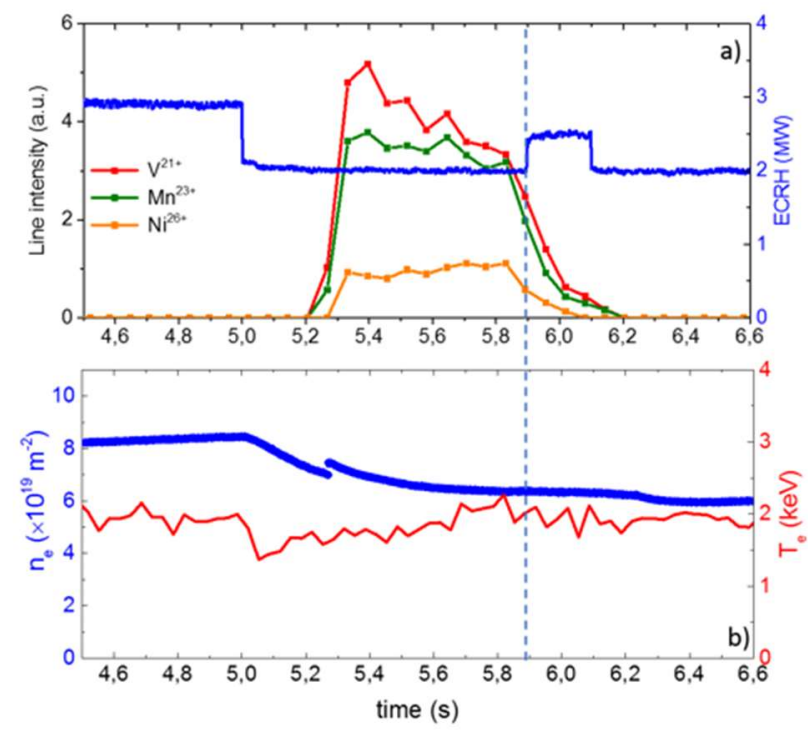

Figure 4. (a) Temporal evolutions of He-like vanadium, manganese and nickel lines observed by the PHA system, compared to ECR heating ; (b) the line-integrated electron density and temperature in 20180919.043 W7-X experimental programme. Dashed line corresponds to the increase of the ECRH.

\section{Summary}

During the OP1.2b of W7-X, several new systems have been commissioned and tested. One of them was TESPEL (Tracer-Encapsulated Solid Pellet) injection system, which allows injecting the impurities locally in the core region of the W7-X plasma. The system is remotely controlled, and the number of tracer impurities injected is well-known, which is highly important for the quantitative study of impurity transport. To check the impact of impurity injection on disturbance of plasma (which should be as small as possible) and investigate the transport of the injected impurities, spectroscopic studies are needed. The pulse height analysis (PHA) system was one of a few W7-X diagnostics, which can observe spectra originated from the injected elements. Consequently, the PHA system has confirmed the proper working of the TESPEL injector. The number of particles is essential in the impurity transport study, since the line emissions from injected impurities should be more intense than the background radiation. In the experiments with the TESPEL injection, medium- and high-Z elements were clearly observed by the PHA system, which suggests that the number of those impurity particles is sufficient for the PHA system.

\section{Acknowledgments}

This work has been carried out within the framework of the EUROfusion Consortium and has received funding from the Euratom research and training programme 2014-2018 and 2019-2020 under grant agreement No 633053. The views and opinions expressed herein do not necessarily reflect those of the European Commission. This scientific work was partly supported by Polish Ministry of Science and Higher Education within the framework of the scientific financial resources in the years 2014-2020 allocated for the realization of the international co-financed project. This work is also supported by JSPS KAKENHI 17KK0121, the Program for Enhancing Research Collaboration (UFEX105), the Young Researchers Supporting Program (UFEX106), the NIFS/NINS under Strategic International Research Exchange Promotion Program 
(UFEX402), the NINS program of Promoting Research by Networking among Institutions (Grant No.01411702) and by Spain's MINCIE FIS2017-89326-F.

\section{References}

[1] M. Hirsch et al. Confinement in Wendelstein 7-X limiter plasmas, Nucl. Fusion 57(8) (2017) 086010.

[2] T. Sunn Petersen et al. Confirmation of the topology of the Wendelstein 7-X magnetic field to better than 1:100,000, Nat. Commun. 7 (2016) 13493.

[3] M. Krychowiak et al., Overview of diagnostic performance and results for the first operation phase in Wendelstein 7-X, Rev. Sci. Instrum. 87 (2016) 11D304.

[4] R.C. Wolf et al., Major results from the first plasma campaign of the Wendelstein 7-X stellarator, Nucl. Fusion 57 (2017) 102020.

[5] T. Klinger et al. Overview of first Wendelstein 7-X high-performance operation, Nucl. Fusion 59 (2019) 112004.

[6] R. Burhenn et al., Transport of Impurity Ions in the Wendelstein 7-AS Stellarator Plasma, AIP Conf. Proc. 812 (2006) 19 https://doi.org/10.1063/1.2168793.

[7] R. Bussiahn et al., Tracer-Encapsulated Solid Pellet (TESPEL) injection system for Wendelstein 7-X, Rev. Sci. Instrum 89, 10K112 (2018).

[8] N. Tamura et al, Transient Transport Studies for Particle and Heat Using Tracer-Encapsulated Solid Pellet lnjection in LHD, J. Plas. Fus. Res. SERIES 5 (2002) 400-403.

[9] S. Sudo et al., Muliple-tracer TESPEL injection for studying impurity behaviour in a magnetically confined plasma, Nucl. Fusion 52 (2012) 063012.

[10] N. Tamura et al., Tracer-Encapsulated Solid Pellet (TESPEL) injection system for the TJ-II stellarator, Rev. Sci. Instrum. 87 (2016) 11D619.

[11] B. Buttenschön et al., Spectroscopic impurity survey in the first operation phase of Wendelstein 7-X, Proceedings of 43rd EPS Conference on Plasma Physics, P4.012, Vol. 40A, ISBN:2-914771-99-1.

[12] A. Langenberg et al., Prospects of X-ray imaging spectrometers for impurity transport: Recent results from the stellarator Wendelstein 7-X, Rev. Sci. Instrum. 89 (2018) 10G101.

[13] M. Kubkowska, B Buttenschön, A. Langenberg and the W7-X team, W7-X plasma diagnostics for impurity transport studies, Prob. Atom. Sci. Tech. 6 Series: Plasma Physics 118 (2018) 312-315.

[14] M. Kubkowska et al. First Results from the Soft X-ray Pulse Height Analysis System on Wendelstein 7-X stellarator, Fusion Eng. Des. 136 (2018) 58-62.

[15] N. Krawczyk et al., Commissioning and first operation of the pulse-height analysis diagnostic on Wendelstein 7-X stellarator, Fusion Eng. Des. 123 (2017) 1006-1010.

[16] M. Kubkowska et al., Plasma impurities observed by a pulse height analysis diagnostic during the divertor campaign of the Wendelstein 7-X stellarator, Rev. Sci. Instrum. 89 (2018) 10F111.

[17] N. Tamura et al., Mitigation of the tracer impurity accumulation by EC heating in the LHD, Plasma Phys. Control Fusion 58 (2016) 114003. 